\title{
Carotid intima-media thickness and plaques in internal carotid artery as surrogate markers of lower limb arterial lesions in Chinese patients with diabetic foot
}

\author{
Mei Zhang (iD, Xiaorong Wen (iD), Chenyun Zhou (iD), Jing Huang (iD), and Ying He (i) \\ Department of Ultrasound, West China Hospital, Sichuan University, Chengdu, Sichuan, China
}

\begin{abstract}
Peripheral arterial disease (PAD) can impair healing of diabetic foot (DF) in patients with diabetes mellitus. To determine whether carotid intima-media thickness (CIMT) can predict lower limb arterial lesions in patients with DF, this cross-sectional study enrolled patients with DF at West China Hospital (China) between January 2012 and December 2015. Ultrasonography was used to measure CIMT, assess the internal carotid arteries (ICA) for plaques, and evaluate lower limb segmental arteries for stenosis. The optimal CIMT cutoff for detecting lower limb PAD was determined by receiver operating characteristic (ROC) curve analysis. Factors associated with PAD were identified by logistic regression analyses. A total of 167 patients (mean age: $69.7 \pm$ 10.3 years; 102 men) were included. Patients with PAD were older and had higher levels of total cholesterol and low-density lipoprotein than patients without PAD $(P<0.05)$. The area under the ROC curve was $0.747(P<0.001)$. At the optimal CIMT cutoff of $0.71 \mathrm{~mm}$, the sensitivity, specificity, positive predictive value, and negative predictive value were $79.65,61.11,81.08$, and $58.93 \%$, respectively. Compared with those without PAD, more patients with PAD had CIMT $\geqslant 0.71 \mathrm{~mm}$ (79.65 vs 38.89\%; $\mathrm{P}<0.001)$ and ICA plaques $(66.37$ vs $11.11 \% ; \mathrm{P}<0.001)$. Multivariate logistic regression revealed that age (odds ratio [OR]: 1.118; 95\% confidence interval [95\%Cl]: $1.056-1.183 ; \mathrm{P}<0.001$ ), ICA plaques (OR: $13.452 ; 95 \% \mathrm{Cl}: 4.450-40.662 ; \mathrm{P}<0.001$ ), and $\mathrm{CIMT} \geqslant 0.71 \mathrm{~mm}$ (OR: $2.802 ; 95 \% \mathrm{Cl}: 1.092-7.188 ; \mathrm{P}=0.032$ ) were associated with PAD.CIMT may be a surrogate marker of PAD in patients with DF.
\end{abstract}

Key words: Diabetes mellitus; Diabetic foot; Carotid artery; Intima-media thickness; Plaque; Peripheral arterial disease

\section{Introduction}

Cardiovascular disease is a leading cause of death in China (1). Type-2 diabetes mellitus (T2DM) is recognized as a major risk factor for cardiovascular disease, and the overall prevalence of T2DM in China has been estimated to be $9.7 \%$, indicating that T2DM is an important public health issue (2). Skin ulceration is a common clinical manifestation of T2DM, occurring in up to $15 \%$ of those with T2DM, and the foot is one of the most frequently affected sites (3). Diabetic foot is a common complication of T2DM that manifests as lower extremity infection, ulcer formation, and/or deep tissue damage secondary to neuropathy and peripheral vascular disease (4). Diabetic foot is an important cause of disability, amputation, and even death in people with T2DM (5). Diabetic foot complications are the most common cause of non-traumatic lower extremity amputation in underdeveloped regions and ethnic minorities (6), and the risk of lower extremity amputation is up to 46 times higher in people with T2DM than in those without T2DM (7).
Locally applied treatments, including appropriate wound care, are key to the management of neuropathic ulcers $(8,9)$. Ulcer healing requires adequate tissue perfusion. However, T2DM is a strong risk factor for occlusive peripheral arterial disease (PAD) (10), and PAD is four times more prevalent in people with T2DM than in those without T2DM (11). People with PAD have a higher risk of all-cause, cardiovascular and coronary artery disease mortality (12). In patients with T2DM, PAD should be suspected if an ulcer fails to heal (13). PAD is present in up to $70 \%$ of patients with diabetic foot $(14,15)$ and is associated with higher incidences of infection and disabling comorbidities (15). Given the association of PAD with poor ulcer healing and amputation, as well as cardiovascular events and premature death, it is important that PAD be diagnosed as early as possible if present in patients with diabetic foot (14). Furthermore, identifying PAD allows assessing whether revascularization surgery or angioplasty is needed to improve blood flow (16).

Correspondence: Xiaorong Wen: <hxwenxiaor@126.com>

Received January 19, 2019 | Accepted May 3, 2019 
However, a formal assessment of lower limb arterial lesions is not always made in patients with diabetic foot.

In 1986, Pignoli and colleagues reported that arterial wall intima-media thickness (IMT) could be measured non-invasively by B-mode ultrasonography (17). Carotid artery IMT (CIMT) is being increasingly used for risk stratification in individuals (18) and as an endpoint in interventional studies (19). A number of investigations have revealed the relationship between IMT and the incidence of cardiac and cerebrovascular events $(20,21)$. Furthermore, CIMT has been reported to be a predictor of multiterritory atherosclerosis (22). However, it is not currently known whether mean CIMT and the presence of internal carotid artery plaques can predict lower limb arterial lesions in patients with diabetic foot. Therefore, in the present study, we investigated whether mean CIMT and the presence of internal carotid artery plaques could be used as markers of lower limb arterial lesions in patients with diabetic foot

\section{Material and Methods}

\section{Study participants}

This cross-sectional study prospectively enrolled consecutive patients with diabetic foot who were admitted to the Diabetic Foot Care Center of West China Hospital (Sichuan University, China) between January 2012 and December 2015. The inclusion criteria were: T2DM diagnosed using internationally recognized criteria (23); a diagnosis of diabetic foot based on clinical history and examination (24); and consent for ultrasonographic measurement of CIMT and evaluation of lower limb arterial stenosis. The exclusion criteria were: previous surgery to the carotid arteries; previous surgery to the lower limbs, including amputation; ultrasonographic measurement of the CIMT failed for whatever reason; and ultrasonographic evaluation of lower limb arterial stenosis was unsuccessful, for whatever reason. The Research Ethics Committee approved this retrospective study and waived written informed consent.

\section{Clinical data}

The following clinical data were obtained for each patient: age, gender, time since diagnosis of T2DM, glycated hemoglobin (HbA1c) level, fasting blood glucose level (FBG), plasma total cholesterol (TC), plasma triglyceride level (TG), plasma low-density lipoprotein level (LDL), systolic blood pressure (SBP), diastolic blood pressure (DBP), smoking status, and alcohol consumption. Hypertension was defined as SBP $\geqslant 140 \mathrm{mmHg}$ or DBP $\geqslant 90 \mathrm{~mm} \mathrm{Hg}$ or current use of antihypertensive medication to treat hypertension (25). Dyslipidemia usually refers to elevated serum high cholesterol, triglycerides, or LDL, and low HDL, commonly known as hyperlipidemia. Hyperlipidemia was defined as total cholesterol $>220 \mathrm{mg} /$ $\mathrm{dL}$ or triglycerides $>170 \mathrm{mg} / \mathrm{dL}$ or $>$ LDL $154 \mathrm{mg} / \mathrm{dL}$.
The Fontaine classification was used to classify the clinical severity of lower limb PAD as grade I: asymptomatic; II: mild-to-moderate intermittent ischemic pain and symptoms, such as intermittent claudication, and pale and cool skin; III: rest pain; or IV: ischemic ulcers or gangrene (26).

\section{Ultrasonographic evaluation of the carotid arteries}

All ultrasound examinations were carried out by the same ultrasonographer, who had more than 15 years of experience in vascular ultrasonography. CIMT was evaluated by high-resolution B-mode ultrasonography using a Philips iU22ultrasound machine equipped with a 3-9 MHz linear array probe (Philips Healthcare, USA). Mean CIMT was automatically measured (by the IU22 system) at a plaque-free site in the far wall of the common carotid artery 1-2 cm below its bifurcation. CIMT was measured on both sides, and the higher value was selected as the value to be used in the analysis.

The bilateral internal carotid arteries were also evaluated for the presence of plaques. A plaque was defined as a maximal IMT $\geqslant 1.5 \mathrm{~mm}$ (27). For the analysis, each patient was classified as either having a plaque (one or more plaques detected in the internal carotid artery on at least one side) or plaque-free (no plaques detected on either side).

\section{Ultrasonographic evaluation of the lower limb arteries}

Ultrasound examination of the lower limb arteries was carried out by an experienced vascular physician using the Philips IU22 ultrasound machine equipped with 5-12 $\mathrm{MHz}$ and 3-9 $\mathrm{MHz}$ probes (which had good resolution and penetration). The common femoral, superficial femoral, popliteal, anterior tibial, posterior tibial, peroneal, and dorsalis pedis arteries were evaluated (Figure 1). The degree of arterial stenosis was categorized as diameter reduced by $<50 \%$, diameter reduced by $50-99 \%$, or complete occlusion (28). The criteria used for classifying the peripheral arterial lesions were based on duplex scanning with spectral waveform analysis: peak systolic velocity was increased by $<100 \%$ relative to the adjacent proximal segment for a reduction in diameter of $0-49 \%$ and was increased by $>100 \%$ relative to the adjacent proximal segment for a reduction in diameter of $50-99 \%$; for complete occlusion, no flow was detected within the imaged arterial segment (35). An overall ultrasound-derived score for lower limb arterial disease was obtained by the piecewise integral method (29): 1 point was recorded for a $0-49 \%$ reduction in diameter, 2 points for a $50-99 \%$ reduction in diameter, and 3 points for complete occlusion. When the same artery showed narrowing in several segments, the score for the segment with the greatest narrowing was used. The arterial segmental scores in each leg were summated, and the higher ultrasonic score of the two legs was used as a measure of lower limb arterial disease. PAD was defined as any segmental artery 

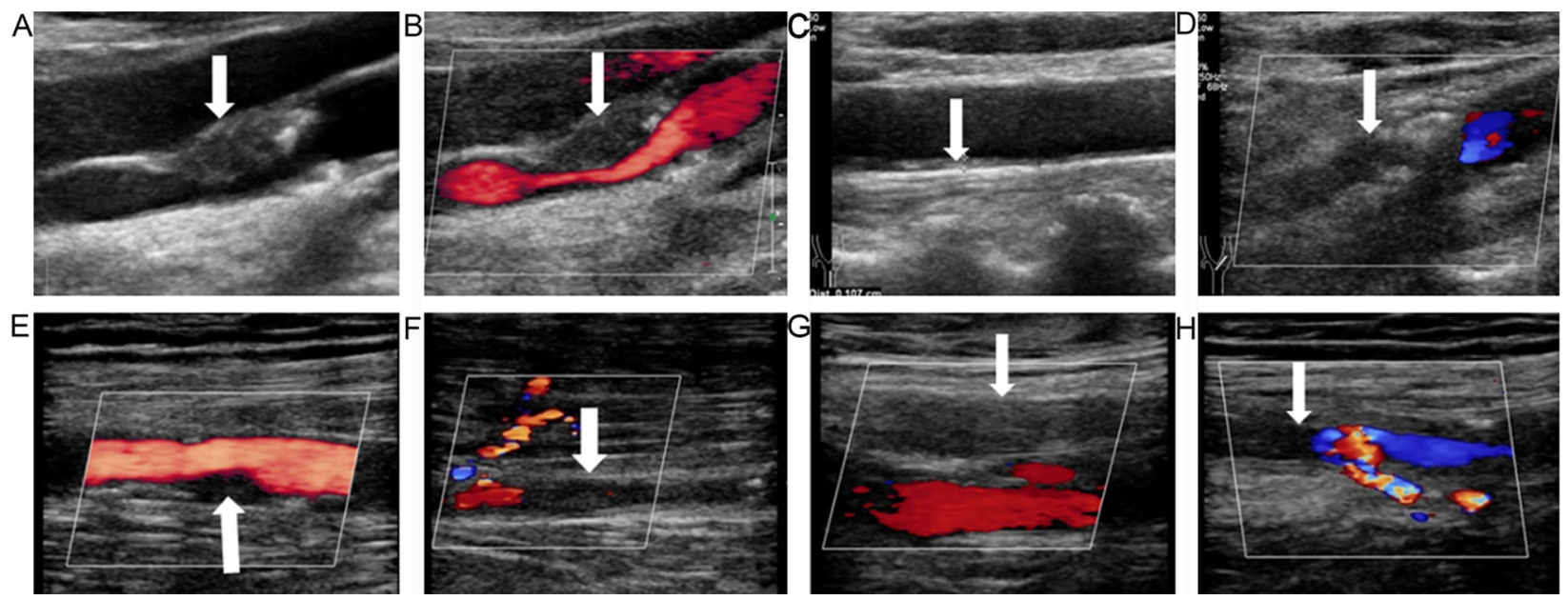

Figure 1. Ultrasonography of the carotid and lower limb arteries of a 74-year-old male patient with diabetic foot, peripheral arterial disease, and plaques in both internal carotid arteries. A, High-resolution gray-scale sonography revealed a plaque (arrow) in the front wall of the right internal carotid artery, and $\mathbf{C}$, an increase in the intima-media thickness of the left common carotid artery (arrow; the measured value was $1.07 \mathrm{~cm}$ ). B, Color Doppler ultrasound showed severe stenosis of the lumen of the right internal carotid artery (arrow; the diameter was reduced by 70-99\%), D, indicated that the left internal carotid artery was occluded (arrow), E, moderate stenosis of the right superficial femoral artery (arrow; the diameter was reduced by 50-99\%), F, segmental occlusion in the anterior tibial artery (arrow) along with a collateral artery, G, occlusion of the left superficial femoral artery (arrow), and $\mathbf{H}$, occlusion of the left superficial femoral artery (arrow) accompanied by a collateral artery. The overall ultrasonic score was 10 points for the right lower limb and 9 points for the left lower limb.

in the lower limbs having a stenosis rate of $50 \%$ or more clinically (30).

\section{Statistical analysis}

Statistical analysis was carried out using SPSS 22.0 (IBM, USA). Continuous variables underwent testing for normality using the Shapiro-Wilk test. Variables are reported as means $\pm S D$ or median (range). Categorical variables are reported as number (\%). Normally distributed continuous data were compared between groups using Student's $t$-test, while Mann-Whitney test was used for non-normally distributed data. Categorical variables were compared between groups with the $\chi^{2}$ test. Receiver operating characteristic (ROC) curve analysis with calculation of the Youden index was used to determine the optimal IMT cutoff for distinguishing between patients with and without $P A D$, and the area under the ROC curve (AUC), sensitivity, specificity, positive predictive value (PPV), and negative predictive value (NPV) were calculated. Univariate and multivariate logistic regression analyses were carried out to identify factors associated with PAD in patients with diabetic foot, with calculation of odds ratios (ORs) and $95 \%$ confidence intervals $(95 \% \mathrm{Cl})$. $\mathrm{P}<0.05$ was taken as statistically significant.

\section{Results}

Clinical characteristics of the study participants

A total of 167 patients (102 men, 65 women) with diabetic foot were included in the analysis (Table 1).
The age of the patients ranged from 38-89years (mean: $69.7 \pm 10.3$ years). The time since diagnosis of T2DM ranged from 0-30 years (mean: $9.8 \pm 6.9$ years), and $\mathrm{HbA} 1 \mathrm{c}$ ranged from $5.7-19.9 \%$ (mean: $8.7 \pm 2.4 \%$ ). According to the Fontaine classification, the severity of the lower limb PAD was graded as level I in 86 patients, level II in 17 patients, level III in 13 patients, and level IV in 51 patients.

Comparison of the clinical characteristics of the study participants between those with PAD $(n=13)$ and those without PAD $(n=54)$ are shown in Table 1. Patients with PAD were significantly older and had higher plasma levels of TC and LDL than patients without PAD $(P<0.05)$. Patients with and without PAD showed no significant differences in gender distribution, time since diagnosis of T2DM, HbA1c, FBG, TG, SBP, DBP, prevalence of smoking, prevalence of alcohol consumption, prevalence of hypertension, or prevalence of hyperlipidemia.

\section{ROC curve analysis of the utility of CIMT for predicting PAD}

ROC curve analysis (Figure 2) of the ability of CIMT to predict PAD found the AUC to be $0.747 \quad(P<0.001)$. Calculation of the Youden index revealed that the optimal cutoff value for CIMT was $0.71 \mathrm{~mm}$. Using this cutoff value, the sensitivity, specificity, PPV, and NPV were determined to be $79.65,61.11,81.08$, and $58.93 \%$, respectively. A CIMT cutoff value of $0.71 \mathrm{~mm}$ was used for the subsequent analyses. 
Table 1. Clinical characteristics of the study participants according to the presence or absence of peripheral arterial disease (PAD).

\begin{tabular}{|c|c|c|c|c|}
\hline & No PAD group $(n=54)$ & PAD group $(n=113)$ & Total $(n=167)$ & $P$ value \\
\hline Age (years) & $62.26 \pm 10.58$ & $73.29 \pm 8.01$ & $69.72 \pm 10.28$ & $<0.001$ \\
\hline \multicolumn{5}{|l|}{ Gender } \\
\hline Female & $19(35.19 \%)$ & $46(40.71 \%)$ & $65(38.92 \%)$ & 0.494 \\
\hline Male & $35(64.81 \%)$ & $67(59.29 \%)$ & $102(61.08 \%)$ & 0.494 \\
\hline Duration of T2DM (years) & $10(0,26)$ & $10(0.17,30)$ & $10(0,30)$ & 0.141 \\
\hline Glycated hemoglobin (\%) & $8.7(5.6,18.7)$ & $8.2(0.8,19.9)$ & $8.3(0.8,19.9)$ & 0.277 \\
\hline Fasting blood glucose (mmol/L) & $8.39(2.95,22.60)$ & $8.05(2.59,45.98)$ & $8.29(2.59,45.98)$ & 0.445 \\
\hline Total cholesterol $(\mathrm{mmol} / \mathrm{L})$ & $3.83(1.90,6.51)$ & $4.07(1.75,9.65)$ & $4.00(1.75,9.65)$ & 0.034 \\
\hline Triglycerides $(\mathrm{mmol} / \mathrm{L})$ & $1.16(0.44,3.78)$ & $1.29(0.45,6.78)$ & $1.27(0.44,6.78)$ & 0.303 \\
\hline Low-density lipoprotein (mmol/L) & $2.07(0.75,4.07)$ & $2.31(0.58,5.81)$ & $2.23(0.58,5.81)$ & 0.025 \\
\hline Systolic blood pressure $(\mathrm{mmHg})$ & $136.0 \pm 17.9$ & $141.3 \pm 22.9$ & $139.6 \pm 21.5$ & 0.142 \\
\hline Diastolic blood pressure $(\mathrm{mmHg})$ & $79.4 \pm 9.9$ & $76.0 \pm 12.5$ & $77.1 \pm 11.8$ & 0.058 \\
\hline \multicolumn{5}{|l|}{ Smoking } \\
\hline No & $31(57.41 \%)$ & $58(51.33 \%)$ & $89(53.29 \%)$ & 0.461 \\
\hline Yes & $23(42.59 \%)$ & $55(48.67 \%)$ & $78(46.71 \%)$ & 0.461 \\
\hline \multicolumn{5}{|l|}{ Alcohol consumption } \\
\hline No & 32 (59.26\%) & $75(66.37 \%)$ & 107 (64.07\%) & 0.370 \\
\hline Yes & $22(40.74 \%)$ & $38(33.63 \%)$ & $60(35.93 \%)$ & 0.370 \\
\hline \multicolumn{5}{|l|}{ Hypertension } \\
\hline No & $27(50.00 \%)$ & $49(43.36 \%)$ & $76(45.51 \%)$ & 0.420 \\
\hline Yes & $27(50.00 \%)$ & $64(56.64 \%)$ & $91(54.49 \%)$ & 0.420 \\
\hline \multicolumn{5}{|l|}{ Hyperlipidemia } \\
\hline No & $45(83.33 \%)$ & $79(69.91 \%)$ & $124(74.25 \%)$ & 0.064 \\
\hline Yes & $9(16.67 \%)$ & $34(30.09 \%)$ & $43(25.75 \%)$ & 0.064 \\
\hline
\end{tabular}

Data are reported as means $\pm S D$, median (range), or number (percentage). T2DM: type-2 diabetes mellitus. Student's $t$-test was used for normally distributed data and Mann-Whitney test was used for non-normally distributed data. Categorical variables were compared between groups with the $\chi^{2}$ test.

\section{CIMT and internal carotid artery plaque status of patients with and without PAD}

The proportion of patients with a CIMT $\geqslant 0.71 \mathrm{~mm}$ was significantly higher in those with PAD $(90 / 113,79.65 \%)$ than in those without PAD (21/54, 38.89\%; $P<0.001)$. Furthermore, internal carotid artery plaques were present in a significantly higher proportion of patients with PAD $(75 / 113,66.37 \%)$ than in those without PAD $(6 / 54$, $11.11 \% ; \mathrm{P}<0.001)$.

\section{Univariate and multivariate logistic regression analyses of factors associated with PAD}

Univariate logistic regression analysis showed that older age, higher TC, higher LDL, presence of internal carotid artery plaques, and CIMT $\geqslant 0.71 \mathrm{~mm}$ were significantly associated with PAD $(P<0.05$; see Table 2 for details). Multivariate logistic regression analysis revealed that older age (OR: $1.118 ; 95 \% \mathrm{Cl}$ : $1.056-$ 1.183; $\mathrm{P}<0.001)$, presence of internal carotid artery plaques (OR: 13.452; 95\%Cl: 4.450-40.662; $\mathrm{P}<0.001$ ), and CIMT $\geqslant 0.71 \mathrm{~mm}$ (OR: 2.802; 95\% Cl: 1.092-7.188; $\mathrm{P}=0.032$ ) were the only factors significantly associated with PAD (Table 3).
Fontaine class, ultrasonic score, and number of lower extremity occluded segments between subgroups based on CIMT and internal carotid artery plaques

When the study participants were divided into two groups based on CIMT, the group with CIMT $\geqslant 0.71 \mathrm{~mm}$ had significantly higher values for the proportion of patients with carotid artery plaques, Fontaine class, ultrasonic score, and number of occluded segments in the lower extremities ( $P \leqslant 0.001$; see Table 4 for details). Furthermore, when the patients were divided into two groups based on the presence or absence of internal carotid artery plaques, the group with internal carotid artery plaques had significantly higher values for Fontaine class, ultrasonic score, and number of occluded segments in the lower extremities $(P<0.001$; Table 5$)$.

\section{Discussion}

A notable finding of the present study was that patients with diabetic foot who had PAD were older and had higher levels of TC and LDL than those who did not have PAD. ROC curve analysis revealed that, at an optimal cutoff value for CIMT of $0.71 \mathrm{~mm}$, the sensitivity, specificity, PPV, 
and NPV for predicting lower limb PAD were 79.65, 61.11, 81.08 , and $58.93 \%$, respectively. Importantly, a higher proportion of patients with PAD had CIMT $\geqslant 0.71 \mathrm{~mm}$ and ICA plaques, compared with patients without PAD. Furthermore, multivariate logistic regression revealed that older age, presence of internal carotid artery plaques, and

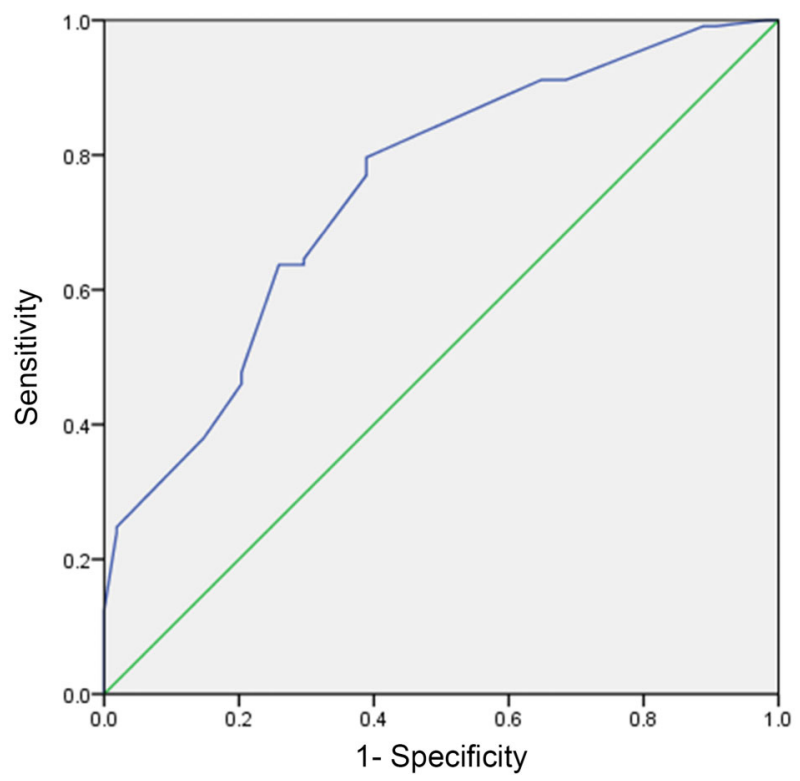

Figure 2. Receiver operating characteristic (ROC) curve analysis of carotid artery intima-media thickness (CIMT) for predicting lower limb peripheral arterial disease. The area under the curve was $0.747(\mathrm{P}<0.001)$, and calculation of the Youden index revealed that the optimal cutoff value for CIMT was $0.71 \mathrm{~mm}$.
CIMT $\geqslant 0.71 \mathrm{~mm}$ were associated with PAD. In addition, patients with CIMT $\geqslant 0.71 \mathrm{~mm}$ or internal carotid artery plaques had higher values for Fontaine class, ultrasonic score, and number of occluded segments in the lower extremities. Taken together, our novel data provide strong evidence that CIMT may be a surrogate marker of lower limb PAD in patients with diabetic foot.

Color duplex ultrasound (DUS) is an important noninvasive tool that can be used as an alternative to digitalsubtraction angiography (DSA) for evaluation of lower extremity arterial disease in patients with diabetic foot. When used for the assessment of lower limb PAD, the agreement between DUS and DSA was reported to be good, irrespective of the severity of the ischemia (31). Furthermore, DUS performed better in the supragenicular arteries than in the infragenicular arteries and compared favorably with DSA in both tibial vessels, particularly in the distal part (31). An important advantage of DUS is that its cost is lower than that of both DSA and magnetic resonance angiography (MRA). The study of Lowery and colleagues (32) concluded that DUS performed extremely well compared with DSA and MRA when used as a preoperative imaging tool in the management of critical lower limb ischemia by endovascular revascularization. Since DSA is an invasive tool, it is perhaps best used for formulating a reasonable preoperative management plan rather than simply for the diagnosis of lower extremity arterial disease. In our study, ultrasonography of the lower limbs of 167 patients with diabetic foot was successful, showing good two-dimensional resolution and sensitive measurement of blood flow. Ultrasound scanning in our study permitted the segmental arteries of the lower limbs to be imaged for stenosis and occlusions, enabling the

Table 2. Univariate logistic regression analysis of factors associated with peripheral arterial disease.

\begin{tabular}{lccr}
\hline Variable & Odds ratio & $95 \% \mathrm{Cl}$ & $\mathrm{P}$ value \\
\hline Gender & 1.265 & $0.645,2.479$ & 0.494 \\
Smoking & 1.278 & $0.665,2.457$ & 0.462 \\
Alcohol consumption & 0.737 & $0.378,1.438$ & 0.371 \\
Hypertension & 1.306 & $0.682,2.503$ & 0.421 \\
Hyperlipidemia & 2.152 & $0.947,4.890$ & 0.067 \\
Presence of internal carotid artery plaque & 15.789 & $6.204,40.183$ & $<0.001$ \\
Age & 1.135 & $1.086,1.186$ & $<0.001$ \\
Time since diagnosis of type-2 diabetes mellitus & 1.046 & $0.995,1.099$ & 0.079 \\
Glycated hemoglobin & 0.904 & $0.793,1.031$ & 0.132 \\
Fasting blood glucose & 1.014 & $0.948,1.084$ & 0.688 \\
Total cholesterol & 1.378 & $1.039,1.828$ & 0.026 \\
Triglycerides & 1.306 & $0.839,2.034$ & 0.237 \\
Low-density lipoprotein & 1.596 & $1.106,2.303$ & 0.012 \\
Systolic blood pressure & 1.012 & $0.996,1.028$ & 0.143 \\
Diastolic blood pressure & 0.975 & $0.948,1.003$ & 0.081 \\
Carotid intima-media thickness $\geqslant 0.71 \mathrm{~mm}$ & 6.149 & $3.013,12.551$ & $<0.001$ \\
\hline
\end{tabular}

95\% Cl: 95\% confidence interval. 
Table 3. Multivariate logistic regression analysis of factors associated with peripheral arterial disease.

\begin{tabular}{lccr}
\hline Variable & Odds ratio & $95 \% \mathrm{Cl}$ & $\mathrm{P}$ value \\
\hline Age & 1.118 & $1.056,1.183$ & $<0.001$ \\
Gender & 1.619 & $0.503,5.214$ & 0.420 \\
Smoking & 1.522 & $0.494,4.694$ & 0.464 \\
Alcohol consumption & 1.414 & $0.447,4.478$ & 0.556 \\
Time since diagnosis of type-2 diabetes mellitus & 1.001 & $0.935,1.073$ & 0.967 \\
Glycated hemoglobin & 1.018 & $0.841,1.232$ & 0.858 \\
Hypertension & 2.024 & $0.783,5.230$ & 0.145 \\
Hyperlipidemia & 1.537 & $0.499,4.733$ & 0.454 \\
Carotid intima-media thickness $\geqslant 0.71 \mathrm{~mm}$ & 2.802 & $1.092,7.188$ & 0.032 \\
Presence of internal carotid artery plaque & 13.452 & $4.450,40.662$ & $<0.001$ \\
\hline
\end{tabular}

95\% Cl: $95 \%$ confidence interval.

Table 4. Comparison of internal carotid artery plaque prevalence, Fontaine class, ultrasonic score, and number of lower extremity occluded segments between subgroups based on carotid intima-media thickness (CIMT).

\begin{tabular}{lccr}
\hline Variable & $\begin{array}{c}\text { CIMT } \begin{array}{c}<0.71 ~ m m \\
(\mathrm{n}=56)\end{array} \\
\text { Internal carotid artery plaques }\end{array}$ & $\begin{array}{c}\mathrm{CIMT} \geqslant 0.71 \mathrm{~mm} \\
(\mathrm{n}=111)\end{array}$ & $\mathrm{P}$ value \\
$\quad$ No & $39(69.64 \%)$ & $47(42.34 \%)$ & 0.001 \\
$\quad$ Yes & $17(30.36 \%)$ & $64(57.66 \%)$ & 0.001 \\
Fontaine class & $1(1-5)$ & $3(1-5)$ & $<0.001$ \\
Ultrasonic score & $7(7-16)$ & $11(7-17)$ & $<0.001$ \\
Number of lower extremity occluded arterial & $0(0-4)$ & $1(0-5)$ & $<0.001$ \\
segments & & & \\
\hline
\end{tabular}

Data are reported as median (range) or number (percentage). $\chi^{2}$ test and Mann-Whitney test were used for analyses.

Table 5. Comparison of Fontaine class, ultrasonic score, and number of lower extremity occluded segments between subgroups based on the presence/absence of internal carotid artery plaques.

\begin{tabular}{lccc}
\hline Variable & $\begin{array}{c}\text { No plaques } \\
(\mathrm{n}=86)\end{array}$ & $\begin{array}{c}\text { With plaques } \\
(\mathrm{n}=81)\end{array}$ & P value \\
\hline Fontaine class & $1(1-5)$ & $4(1-5)$ & $<0.001$ \\
Ultrasonic score & $7(7-17)$ & $12(7-16)$ & $<0.001$ \\
Number of lower extremity occluded arterial & $0(0-5)$ & $2(0-4)$ & $<0.001$ \\
segments & & & \\
\hline
\end{tabular}

Data are reported as median (range). Mann-Whitney test was used for analyses.

overall severity of the PAD to be graded using the piecewise integral method based on the degree of arterial stenosis. Therefore, DUS of the lower limbs represents a non-invasive and cost-effective method of assessing lower limb PAD in patients with diabetic foot.

CIMT is a surrogate marker for early atherosclerosis that can be measured relatively simply and non-invasively with high-resolution ultrasonography. CIMT is a recognized marker of coronary and cerebral artery atherosclerosis (20-22). Two methods may be used to acquire CIMT: one method involves measurements of maximal CIMT in the near and far walls of the three main segments of the carotid arteries (common carotid, bifurcation, and internal carotid); the other method involves an automated measurement of CIMT, which is restricted to the far wall of the distal common carotid artery at a plaque-free site. 
Computerized measurements of CIMT are superior to manual measurements in terms of precision and reproducibility, with a difference of only $\sim 3 \%$ between two successive automated measurements (33). Therefore, we adopted automated measurement of CIMT in the far wall of the distal common carotid artery at a plaque-free site. Our results showed that a CIMT cutoff value of $0.71 \mathrm{~mm}$ was able to predict lower limb PAD with a sensitivity of nearly $80 \%$ and a specificity of over $60 \%$. Furthermore, nearly $80 \%$ of patients with PAD had CIMT $\geqslant 0.71 \mathrm{~mm}$, compared with less than $40 \%$ of patients without PAD, and CIMT $\geqslant 0.71 \mathrm{~mm}$ was found to be an independent predictor of PAD in multivariate analysis. In addition, the group of patients with CIMT $\geqslant 0.71 \mathrm{~mm}$ had a higher Fontaine class, higher ultrasonic score for the lower extremities, and greater number of occluded arterial segments in the lower limbs, indicating more severe lower extremity arterial disease than patients with CIMT $<0.71 \mathrm{~mm}$. These findings indicate that CIMT $\geqslant 0.71 \mathrm{~mm}$ may be a useful surrogate marker for lower limb PAD in patients with diabetic foot. To the best of our knowledge, no previous studies have explored the relationship between CIMT and lower extremity PAD specifically in patients with diabetic foot. However, CIMT is a strong predictor of future vascular events (34), and lower extremity arterial atherosclerosis has been reported to progress more rapidly with increasing CIMT (35). Furthermore, two studies found that patients with PAD had a significantly higher CIMT than age-matched healthy controls: $0.8 \pm 0.2 \mathrm{~mm}$ vs $0.6 \pm$ $0.1 \mathrm{~mm}(36)$ and $0.85 \pm 0.35 \mathrm{~mm}$ vs $0.59 \pm 0.23 \mathrm{~mm}$ (37). Our finding that the optimal cutoff value for CIMT was $0.71 \mathrm{~mm}$ is in good agreement with these observations. The Rotterdam study determined that the OR for lower extremity arterial disease was 3.4 for people with a CIMT $\geqslant 0.89 \mathrm{~mm}$ (corresponding to the upper quintile) (38). Although the CIMT value in this previous study was higher than that used in ours, it should be noted that the Rotterdam study did not perform ROC curve analysis to determine an optimal cutoff value and included a study population that was not limited to patients with T2DM and diabetic foot.

There is little published data on the correlation between carotid plaques and lower limb arterial disease in patients with diabetic foot. Our study found that internal carotid artery plaques were present in a much higher

\section{References}

1. He J, Gu D, Wu X, Reynolds K, Duan X, Yao C, et al. Major causes of death among men and women in China. N Engl $J$ Med 2005; 353: 1124-1134, doi: 10.1056/NEJMsa050467.

2. Yang W, Lu J, Weng J, Jia W, Ji L, Xiao J, et al. Prevalence of diabetes among men and women in China. N Engl J Med 2010; 362: 1090-1101, doi: 10.1056/NEJMoa0908292.

3. Pendsey SP. Understanding diabetic foot. Int J Diabetes Dev Ctries2 2010; 30: 75-79, doi: 10.4103/0973-3930.62596. proportion of patients with PAD than in those without PAD (66 vs 11\%), and the presence of plaques in the internal carotid arteries was an independent predictor of lower limb PAD in multivariate analysis. Furthermore, patients with internal carotid artery plaques had higher values for Fontaine class, ultrasonic score, and number of occluded segments in the lower extremities. These results suggest that, in patients with diabetic foot, the existence of internal carotid artery plaques is an indicator of more serious PAD in the lower extremities. Consistent with our observations, it has been reported that carotid artery plaques are much more common in patients with PAD than in healthy controls (36). The carotid artery is a good window for observing atherosclerosis, which is a systemic disease. An increase in CIMT is considered an early marker of atherosclerosis, but plaques in the carotid arteries are not early arterial lesions. Thus, by the time that plaques emerge in the carotid arteries, it is more likely that atherosclerosis of the arteries in the lower limbs will have occurred. Indeed, it has been reported that carotid plaques are independent predictors of coronary heart disease and can improve risk prediction in older adults (39).

This study has some limitations. First, this was a single-center study with quite a small sample size, so the findings cannot be generalized. Second, this was a crosssectional study rather than a longitudinal study, so the utility of CIMT or internal carotid artery plaques in the prediction of clinical outcomes such as foot ulcer healing was not examined. Third, other (non-ultrasonographic) methods of assessing lower extremity arterial disease, such as DSA and MRA, were not used for comparison. Additional studies are needed to further examine the clinical utility of CIMT and internal carotid artery plaques for the evaluation of lower limb PAD and prediction of ulcer healing in patients with diabetic foot.

CIMT and the presence of internal carotid artery plaques may be useful clinical markers of lower limb arterial lesions in patients with diabetic foot.

\section{Acknowledgments}

The authors are grateful to all the study participants and staff at West China Hospital who provided their assistance during the undertaking of this research.
4. Apelqvist J. Diagnostics and treatment of the diabetic foot. Endocrine 2012; 41: 384-397, doi: 10.1007/s12020-0129619-x.

5. Dohmen A, Eder S, Euringer W, Zeller T, Beyersdorf F. Chronic critical limb ischemia. Dtsch Arztebllnt 2012; 109: 95-101, doi: 10.3238/arztebl.2012.0095.

6. Lavery LA, Ashry HR, van Houtum W, Pugh JA, Harkless LB, Basu S. Variation in the incidence and proportion of 
diabetes-related amputations in minorities. Diabetes Care 1996; 19: 48-52, doi: 10.2337/diacare.19.1.48.

7. Armstrong DG, Lavery LA, Quebedeaux TL, Walker SC. Surgical morbidity and the risk of amputation due to infected puncture wounds in diabetic versus nondiabetic adults. South Med J 1997; 90: 384-389, doi: 10.1097/00007611199704000-00004.

8. Kavitha KV, Tiwari S, Purandare VB, Khedkar S, Bhosale SS, Unnikrishnan AG. Choice of wound care in diabetic foot ulcer: a practical approach. World J Diabetes 2014; 5: 546556, doi: 10.4239/wjd.v5.i4.546.

9. Game FL, Hinchliffe RJ, Apelqvist J, Armstrong DG, Bakker $\mathrm{K}$, Hartemann A, et al. A systematic review of interventions to enhance the healing of chronic ulcers of the foot in diabetes. Diabetes Metab Res Rev 2012; 28 (Suppl 1): 119141, doi: 10.1002/dmrr.2246.

10. Criqui $\mathrm{MH}$, Aboyans V. Epidemiology of peripheral artery disease. Circ Res 2015; 116: 1509-1526, doi: 10.1161/ CIRCRESAHA.116.303849.

11. Kannel WB, McGee DL. Diabetes and glucose tolerance as risk factors for cardiovascular disease: the Framingham study. Diabetes Care 1979; 2: 120-126, doi: 10.2337/diacare. 2.2.120.

12. Aronow WS. Office management of peripheral arterial disease. Am J Med 2010; 123: 790-792, doi: 10.1016/j. amjmed.2010.03.017.

13. Armstrong DG, Lavery LA. Diabetic foot ulcers: prevention, diagnosis and classification. Am Fam Physician 1998; 57: 1325-1332, 1337-1328.

14. Brownrigg JR, Schaper NC, Hinchliffe RJ. Diagnosis and assessment of peripheral arterial disease in the diabetic foot. Diabet Med 2015; 32: 738-747, doi: 10.1111/dme.12749.

15. Hao D, Hu C, Zhang T, Feng G, Chai J, Li T. Contribution of infection and peripheral artery disease to severity of diabetic foot ulcers in Chinese patients. Int J Clin Pract 2014; 68: 1161-1164, doi: $10.1111 /$ ijcp.12440.

16. Forsythe RO, Brownrigg J, Hinchliffe RJ. Peripheral arterial disease and revascularization of the diabetic foot. Diabetes Obes Metab 2015; 17: 435-444, doi: 10.1111/ dom.12422.

17. Pignoli P, Tremoli E, Poli A, Oreste P, Paoletti R. Intimal plus medial thickness of the arterial wall: a direct measurement with ultrasound imaging. Circulation 1986; 74: 1399-1406, doi: 10.1161/01.CIR.74.6.1399.

18. Naqvi TZ, Lee MS. Carotid intima-media thickness and plaque in cardiovascular risk assessment. JACC CardiovasC Imaging 2014; 7: 1025-1038, doi: 10.1016/j.jcmg.2013. 11.014

19. Peters SA, Grobbee DE, Bots ML. Carotid intima-media thickness: a suitable alternative for cardiovascular risk as outcome? Eur J Cardiovasc Prev Rehabil 2011; 18: 167174, doi: 10.1177/1741826710389400.

20. O'Leary DH, Polak JF, Kronmal RA, Manolio TA, Burke GL, Wolfson SK Jr. Carotid-artery intima and media thickness as a risk factor for myocardial infarction and stroke in older adults. Cardiovascular Health Study Collaborative Research Group. N Engl J Med 1999; 340: 14-22, doi: 10.1056/ NEJM199901073400103.

21. Hollander M, Hak AE, Koudstaal PJ, Bots ML, Grobbee DE, Hofman A, et al. Comparison between measures of atherosclerosis and risk of stroke: the Rotterdam Study. Stroke
2003; 34: 2367-2372, doi: 10.1161/01.STR.0000091393. 32060.0E

22. Kablak-Ziembicka A, Przewlocki T, Pieniazek P, Musialek P, Sokolowski $A$, Drwila $R$, et al. The role of carotid intima-media thickness assessment in cardiovascular risk evaluation in patients with polyvascular atherosclerosis. Atherosclerosis 2010; 209: 125-130, doi: 10.1016/j.athero sclerosis.2009.08.019.

23. American Diabetes Association. Diagnosis and classification of diabetes mellitus. Diabetes Care 2010; 33 (Suppl 1): S62-S69, doi: 10.2337/dc10-S062.

24. Doupis J, Veves A. Classification, diagnosis, and treatment of diabetic foot ulcers. Wounds 2008; 20: 117-126.

25. Chobanian AV, Bakris GL, Black HR, Cushman WC, Green LA, Izzo JL Jr, et al. Seventh report of the Joint National Committee on prevention, detection, evaluation, and treatment of high blood pressure. Hypertension 2003; 42: 12061252, doi: 10.1161/01.HYP.0000107251.49515.c2.

26. Fontaine R, Kim M, Kieny R. Surgical treatment of peripheral circulation disorders. Helv Chir Acta 1954; 21: 499-533.

27. Touboul PJ, Hennerici MG, Meairs S, Adams H, Amarenco P, Bornstein $\mathrm{N}$, et al. Mannheim carotid intima-media thickness and plaque consensus (2004-2006-2011). An update on behalf of the advisory board of the 3rd, 4th and 5th Watching the Risk Symposia, at the 13th, 15th and 20th European Stroke Conferences, Mannheim, Germany, 2004, Brussels, Belgium, 2006, and Hamburg, Germany, 2011. Cerebrovasc Dis 2012; 34: 290-296, doi: 10.1159/000343145.

28. Zierler RE. Ultrasound assessment of lower extremity arteries. In: Zwiebel WJ, Pellerito JS, eds. Introduction to Vascular Ultrasonography, 5th edn. Philadelphia: Saunders; 2005. p 348-349.

29. van der Feen C, Neijens FS, Kanters SD, Mali WP, Stolk RP, Banga JD. Angiographic distribution of lower extremity atherosclerosis in patients with and without diabetes. Diabet Med 2002; 19: 366-370, doi: 10.1046/j.1464-5491.2002. 00642.x.

30. Ogata H, Kumata-Maeta C, Shishido K, Mizobuchi M, Yamamoto M, Koiwa F, et al. Detection of peripheral artery disease by duplex ultrasonography among hemodialysis patients. Clin J Am SocNephrol 2010; 5: 2199-2206, doi: 10.2215/CJN.09451209.

31. Eiberg JP, Gronvall Rasmussen JB, Hansen MA, Schroeder TV. Duplex ultrasound scanning of peripheral arterial disease of the lower limb. Eur J Vasc Endovasc Surg 2010; 40: 507-512, doi: 10.1016/j.ejvs.2010.06.002.

32. Lowery AJ, Hynes N, Manning BJ, Mahendran M, Tawfik S, Sultan S. A prospective feasibility study of duplex ultrasound arterial mapping, digital-subtraction angiography, and magnetic resonance angiography in management of critical lower limb ischemia by endovascular revascularization. Ann Vasc Surg 2007; 21: 443-451, doi: 10.1016/j.avsg.2006. 08.005

33. Graf S, Gariepy J, Massonneau M, Armentano RL, Mansour $S$, Barra JG, et al. Experimental and clinical validation of arterial diameter waveform and intimal media thickness obtained from B-mode ultrasound image processing. Ultrasound Med Biol 1999; 25: 1353-1363, doi: 10.1016/S03015629(99)00089-7.

34. Lorenz MW, Markus HS, Bots ML, Rosvall M, Sitzer M. Prediction of clinical cardiovascular events with carotid 
intima-media thickness: a systematic review and metaanalysis. Circulation 2007; 115: 459-467, doi: 10.1161/ CIRCULATIONAHA.106.628875.

35. Nosenko NS, Nosenko EM, Dadova LV, Sidorenko BA. The risk factors and predictors of the clinically significant progression of atherosclerosis in patients with chronic lower extremity ischemia. Ter Arkh 2010; 82: 56-60.

36. Poredos P, Golob M, Jensterle M. Interrelationship between peripheral arterial occlusive disease, carotid atherosclerosis and flow mediated dilation of the brachial artery. Int Angiol 2003; 22: 83-87.

37. Cheng KS, Tiwari A, Baker CR, Morris R, Hamilton G, Seifalian AM. Impaired carotid and femoral viscoelastic properties and elevated intima-media thickness in peripheral vascular disease. Atherosclerosis 2002; 164: 113-120, doi: 10.1016/S0021-9150(02)00042-4.

38. Bots ML, Hofman A, Grobbee DE. Common carotid intimamedia thickness and lower extremity arterial atherosclerosis. The Rotterdam Study. Arterioscler Thromb 1994; 14: 18851891, doi: 10.1161/01.ATV.14.12.1885.

39. Plichart M, Celermajer DS, Zureik M, Helmer C, Jouven X, Ritchie K, et al. Carotid intima-media thickness in plaquefree site, carotid plaques and coronary heart disease risk prediction in older adults. The Three-City Study. Atherosclerosis 2011; 219: 917-924, doi: 10.1016/j.atheroscle rosis.2011.09.024. 